\title{
Panel Device
}

National Cancer Institute

\section{Source}

National Cancer Institute. Panel Device. NCI Thesaurus. Code C50096.

A rigid sheet that forms a surface of a device or component. 